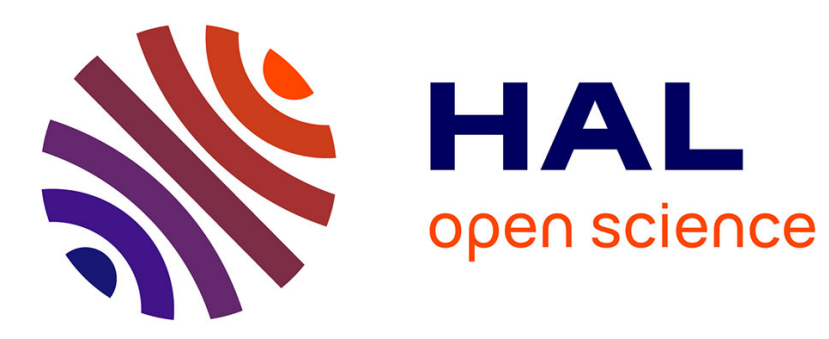

\title{
Une masse nasale atypique
}

\author{
E. Drapier, M. Makeieff, X. Dubernard
}

\section{To cite this version:}

E. Drapier, M. Makeieff, X. Dubernard. Une masse nasale atypique. Annales françaises d'Oto-rhinolaryngologie et de Pathologie Cervico-faciale, 2019, 136, pp.50 - 52. 10.1016/j.aforl.2018.08.003 . hal-03486556

\section{HAL Id: hal-03486556 https://hal.science/hal-03486556}

Submitted on 20 Dec 2021

HAL is a multi-disciplinary open access archive for the deposit and dissemination of scientific research documents, whether they are published or not. The documents may come from teaching and research institutions in France or abroad, or from public or private research centers.
L'archive ouverte pluridisciplinaire HAL, est destinée au dépôt et à la diffusion de documents scientifiques de niveau recherche, publiés ou non, émanant des établissements d'enseignement et de recherche français ou étrangers, des laboratoires publics ou privés.

\section{다)(1) $(5$}

Distributed under a Creative Commons Attribution - NonCommercial| 4.0 International 


\title{
Une masse nasale atypique.
}

\author{
E. Drapier ${ }^{1}$, M. Makeieff ${ }^{1}$, X. Dubernard ${ }^{1 *}$
}

${ }^{1}$ Service d'oto-rhino-laryngologie, CHU de Reims, rue du General-Koening, 51100 Reims, France

- Service d'ORL du CHU de Reims, Hôpital Robert Debré, Rue Cognac Jay, France

*-Auteur correspondant

e-mail : xdubernard@chu-reims.fr_(X Dubernard)

$-\mathrm{Tel}: 0326787714$ 
Mots clef: angioleïomyome, fosse nasale, tumeur bénigne

\section{Description:}

Un patient de 60 ans se présente en consultation pour épistaxis unilatérale gauche chronique depuis au moins 3 mois. Il n'a jamais été exposé à la poussière de bois, ne présente ni diabète, ni hypertension artérielle. Il a récemment été pris en charge pour un cancer papillaire thyroïdien par thyroïdectomie et curage cervical suivi d'une ira thérapie à l'iode 131.

Sur le plan fonctionnel, il n'existe ni douleur, ni obstruction nasale, ni anosmie ou rhinorrhée. L'examen clinique retrouve une lésion d'aspect ovoïde, bien limitée, hyper vascularisée, saignant au contact, sans sécrétion purulente et pédiculée sous le cornet moyen (figure 1). On ne note aucun trouble ophtalmologique ou nerveux notamment sur les nerfs crâniens et en particulier sur le nerf trigéminal. Un bilan d'imagerie associant un scanner injecté et une imagerie par résonnance magnétique (IRM) est réalisé (figure 2).

Quel est votre diagnostic? 
Réponse: Un angioléïomyome de la fosse nasale gauche

Décrit initialement par Maesaka et al. dans cette localisation, l'angioléïomyome est une tumeur rare de la fosse nasale.[1] Il représente moins de $1 \%$ de tous les angioleïomyomes du corps humain [2,3]. Se développant à partir des fibres musculaires lisses, ses localisations préférentielles sont les organes génitaux féminins (95\%), la peau (3\%) et le tractus digestif $(1,5 \%)$ [2]. Différentes hypothèses attribuent son origine dans la fosse nasale aux cellules musculaires lisses des parois des vaisseaux sanguins, des muscles érecteurs des follicules pileux autour des glandes sudoripares ou d'un tissu embryonnaire remanié et indifférencié. $[2,3]$. Sa présentation clinique reste peu spécifique et se manifeste surtout par des épistaxis à répétition et une obstruction nasale. L'incidence de développement la plus élevée se situe entre la quatrième et la sixième décennie [2]. Il apparait souvent comme une masse bien limitée et très vascularisée ce qui contre indique toute biopsie en consultation. Il peut prendre naissance sur l'ensemble de la fosse nasale avec une prédominance pour le cornet inférieur, le septum et le vestibule nasal. Chez ce patient, la lésion était localisée sous l'auvent du cornet moyen. L'examen clinique ne retrouve aucune atteinte ophtalmologique ou cérébrale, cette lésion n'ayant qu'un potentiel de croissance local sans envahissement des structures adjacentes. Le scanner injecté et l'imagerie par résonnance magnétique sont essentiels pour caractériser la masse et éliminer les diagnostics différentiels.

Typiquement le scanner injecté retrouve une lésion iso dense aux muscles, bien limitée, vascularisée tout en restant à distance de l'artère sphéno-palatine permettant d'exclure le diagnostic d'angiofibrome naso-pharyngien. Il n'existe pas de lyse osseuse et selon le volume, le septum nasal peut être refoulé. L'IRM injectée retrouve classiquement une lésion en iso signal T1 tout comme les muscles, un hyper signal T2 et un rehaussement homogène et intense après injection de gadolinium [4].

Les diagnostics différentiels à évoquer sont en premier lieu les tumeurs malignes nasosinusiennes, l'angiomyolipome, le botriomycome, l’hémangiome, le léiomyosarcome, le fibromyome.

Les tumeurs malignes des fosses nasales sont de natures histologiques variées et parfois liées à des facteurs de risque (exposition aux poussières de bois). De diagnostic parfois tardif, l'examen clinique peut retrouver des atteintes neurologiques (hypoesthésie et/ou névralgie faciale par atteinte trigéminale), ophtalmologiques (diplopie, exophtalmie). Sur l'imagerie et à la différence de l'angioléïomyome, ces lésions sont mal limitées et envahissent les structures adjacentes (cavité orbitaire) par lyse osseuse tout en s'associant de façon fréquente à des calcifications intra tumorales. Le rehaussement au gadolinium est bien souvent hétérogène.

L'angiomyolipome, tumeur bénigne cellulo-graisseuse, ne peut pas être évoqué chez ce patient devant l'absence d'hypersignal T1 à l'IRM.

Le botriomycome est une étiologie fréquente, lui aussi tissulaire et très vascularisé survenant souvent chez les patients après la cinquième décennie. Seule l'analyse 
anatomopathologique permet d'en affirmer le diagnostic, la présentation clinique étant très semblable à celle de l'angioleïomyome.

Dans ce contexte, l'étude anatomopathologique prend toute sa valeur puisqu'elle est la seule à pouvoir exclure avec certitude les diagnostics différentiels. La biopsie première au bloc opératoire ou l'exérèse chirurgicale initiale dans le cas où la lésion est bien limitée, est donc impérative. Dans le cas où le diagnostic d'angioléïomyome est porté, comme chez notre patient, l'analyse anatomopathologique retrouve une prolifération vasculaire et musculaire lisse hyperplasique organoïde péri vasculaire. Cette formation péri-vasculaire concentrique est constituée de grandes cellules fusiformes à cytoplasme éosinophile ou clair avec un rapport nucléo-cytoplasmique bas. L'étude immuno-histochimique de cette lésion montre la présence d'un phénotype musculaire lisse mature avec expression de marqueurs positifs comme la caldesmone et négatifs comme le HMB45[5]. On remarque que la présence de moins de 3\% de tissu adipeux mature exclut le diagnostic d'angiomyolipome. L'absence d'atypie nucléaire, de noyaux tumoraux en forme de cigares disposés en faisceaux entrelacés, et l'absence de nombreuses mitoses éliminent aussi le diagnostic de leïomyosarcome chez ce patient.[3]

Une fois opéré et reséqué en totalité, l'angioléïomyome a un pronostic excellent. Son traitement est exclusivement chirurgical et le pourcentage de récidive est très faible. Nous conseillons une surveillance clinique à 6 mois puis à 1 an, associée un contrôle radiologique afin de ne pas méconnaître ce risque.

Les tumeurs des fosses nasales présentent une grande variabilité d'histologies bénignes et malignes. Ces dernières doivent toujours être caractérisées sur le plan radiologique et anatomopathologique. L'angioleïomyome est une forme bénigne particulière dont il faut pouvoir évoquer le diagnostic même devant ces localisations rares.

\section{Conflit d'intérêt : aucun}




\section{Références:}

[1] Maesaka A, Keyaki Y, Nakahashi T. Nasal angioleiomyoma and leiomyosarcoma: report of 2 cases. Otology. 1966;12:42-47.

[2] Arruda MM, Monteiro DY, Fernandes AM, Menegatti V, Thomazzi E, Hubner RA, et al. Angioleiomyoma of the Nasal Cavity. Int Arch Otorhinolaryngol. oct 2014;18(4):409-11.

[3] Mathieu T, Verbruggen A, Goovaerts G, Declau F. Vascular leiomyoma of the nasal cavity: case report and literature review. Eur Arch Otorhinolaryngol. janv 2017;274(1):57983.

[4] Yang BT, Wang ZC, Xian JF, Hao DP, Chen QH. Leiomyoma of the sinonasal cavity: CT and MRI findings. Clin Radiol. déc 2009;64(12):1203-9.

[5] Agaimy A, Michal M, Thompson LDR, Michal M. Angioleiomyoma of the Sinonasal Tract: Analysis of 16 Cases and Review of the Literature. Head Neck Pathol. 6 juin 2015;9(4):463-73. 
Figure 1 : Vue endoscopique fosse nasale gauche au niveau du méat moyen.

Figure 2: Coupes axiales en imagerie par résonnance magnétique, séquences T1, T2 et T1 gadolinium. 


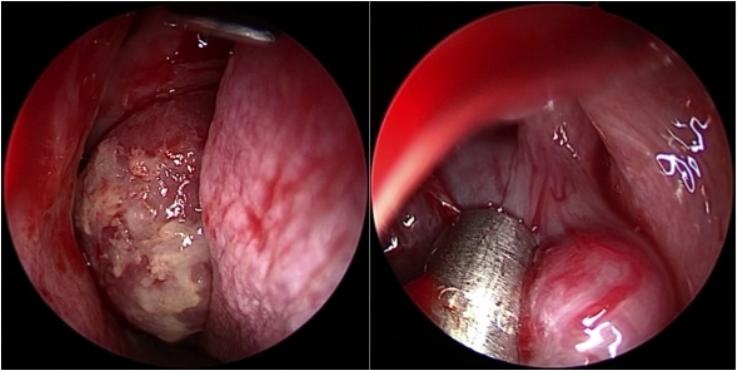


